Received: November 21, 2017

Revision received: March 25, 2018

\title{
Effect Channels of Education Level and Cognitive Skill on Economic Growth
}

\author{
Lanfang Deng ${ }^{1}$ \\ Hunan University
}

\author{
Yulong Zhao ${ }^{2}$ \\ Zhengzhou Central Sub-branch of China \\ People Bank
}

\begin{abstract}
This paper aims to disclose the direct and indirect effect channels of education level and cognitive skill on economic growth. For this purpose, the effective education was introduced to analyse the provincial panel data in 1996 2012 against three levels of education in China: compulsory education, senior high school education and higher education. The education level and cognitive skill were treated as components of heterogeneous human capital. The empirical analysis shows that: On the compulsory education level, neither educated populated nor cognitive skill has any direct or indirect impact on economic growth. On the level of senior high school education, the education level has a significant positive effect on economic growth indirectly through innovation imitation. On the higher education level, the human capital exerts a greater positive effect on economic growth directly as a production input than that of compulsory and senior high school educations. The research findings shed new light on the improvement of economic growth through human capital accumulation at different education levels.
\end{abstract}

\section{Keywords}

Cognitive Skill • Education Level • Technology Innovation • Innovation Imitation • Economic Growth

\footnotetext{
${ }^{1}$ Correspondence to: Centre for Economics, Finance and Management Studies, Hunan University, Changsha 410082, China. Email: denglanfang1987227@126.com

${ }^{2}$ Zhengzhou Central Sub-branch of China People Bank, Zhengzhou 450003, China. Email: xuezhong215@ foxmail.com
} 
It is well known that human capital has a positive impact on economic growth. However, most researchers have only measured the impact of human capital by education level, ignoring many other influencing factors. Thus, this paper introduces another influencing factor: cognitive skill (Behrman and Birdsall 1983), and estimates the contribution of heterogeneous cognitive skill to GDP growth through the human capital.

Many scholars have explored the relationship between cognitive skill and human capital. Based on human capital theory, (Hanushek and Woessmann, 2008) held that cognitive skill is reflected by the quality, not the quantity, of education. Theodore (Breton, 2011) argued that education level is a major influencing factor of GDP growth, because mean education level can explain per-capita GDP differences among countries better than mean test score. Some scholars have noticed the heterogeneous distribution of human capital and attributed the misallocation to various causes, including but not limited to financing frictions (Buera and Shin, 2009; Midrigan and Xu, 2014), implicit taxes (Restuccia and Rogerson, 2008; Hsieh and Klenow, 2009), and the difference in on-job training/education between enterprises (Jovanovic, 2014). These studies have shown that inequality always lingers on the road to balanced growth.

The heterogeneous cognitive skill has often been associated with innovation. Since (Griliches, 1979) put forward the concept of knowledge production function, much research has been done to disclose the correlation between knowledge production and innovation. (Lucas, 1988) pointed out that human capital, which is closely intertwined with cognitive skill, affecting the efficiency of regional innovation. By contrast, there are only a few reports on the indirect impact of human capital on economic growth through innovation (Paganetto and Scandizzo, 2003). Using provincial data in China, (Qian, 2010) estimated the effect of human capital on innovation by spatial regression models, and confirmed the significant impact of human capital on innovation: the education level is positively correlated with knowledge acquisition and innovation.

In general, the existing studies on the relationship between human capital and economic growth mostly focus on the education level Nevertheless, (Barro, 1991) carried out regression analysis on the per-capita GDP growth rate from 1960 to 1985, and discovered the positive impacts of primary and secondary enrolment rates on economic growth rate. (Hanushek and Kimko, 2000) pointed out that the economic growth in both developed and developing countries can be better explained by variation in education quality than that in education. (Hu and Hibel, 2015) explored the heterogeneous returns to higher education for people at different income levels, revealing that the effect of college education is the most obvious for those at the top of the income ladder.

Most scholars have agreed that education has a positive impact on GDP growth, thanks to its direct effect on the accumulation of human capital. Some of them have measured the impact of human capital by heterogeneous cognitive skill and other components of human capital, in addition to education. (Hanushek and Kimko, 2000) are the first to weigh the education quality across different countries against the cognitive skill of students in various international tests on math and science. Since some indices are unavailable, (Zhan, 2010) measured the education quality by the test score and student-teacher ratio. All these studies have shown the close correlation between education quality and cognitive skill.

In light of the above, this paper differentiates the cognitive skill and educated population among different education levels in China, i.e. compulsory education, senior high school education and higher education, and 
explains the channels through which human capital accumulation affect economic growth. The remainder of this paper is organized as follows: Section 2 describes the variables in our model and the data sources; Section 3 explains the methodology; Section 4 analyses the main results; Section 5 wraps up this paper with some meaningful conclusions.

\section{Data Sources and Variables}

All the data were collected from China Statistical Yearbooks and China Labour Statistical Yearbooks from 1996 to 2012. These data cover 30 provinces, municipalities and autonomous regions in Chinese mainland. Note that Sichuan Province and Chongqing Municipality are treated as one entity in these sources. The main variables of our research are shown in Table 1 below.

In the table above, the student-teacher ratio is a proxy variable of cognitive skill (Barro, 1991; Bils and Klenow, 2000; Kanushek and Kimko, 2000). This ratio is positively correlated with the regional cognitive outcome. The explanatory variables are the total labour force and the total fixed capital, and the dependent variable is the GDP.

In addition, the effective education (Behrman and Birdsall 1983) was introduced to measure the cognitive skill. The human capital was divided into education quantity (education level) and education quality (cognitive skill). Then, the human capital function was redefined by the variables of effective education.

Table 1

Description of the Main Variables

\begin{tabular}{lccccc}
\hline Variable & Obs & Mean & SD & Min & Max \\
\hline GDP (million) & 510 & 7611.3 & 9018 & 64.8 & 57067.9 \\
\hline Labor (thousand) & 510 & 3083.6 & 2054.5 & 148.6 & 8476.8 \\
\hline Investment (thousand) & 510 & 3922.4 & 5120.7 & 29.4 & 31256 \\
\hline Compulsory education ratio (\%) & 510 & 41.4 & 10.1 & 2.6 & 59.6 \\
\hline Senior high school education ratio (\%) & 510 & 13.8 & 5.7 & 0.1 & 36.1 \\
\hline Higher education ratio (\%) & 510 & 8.2 & 6.7 & 0 & 53.6 \\
\hline Student-teacher Ratio-Compulsory (\%) & 510 & 16.69 & 2.99 & 9.6 & 25.6 \\
\hline Student-teacher Ratio- Senior high(\%) & 510 & 16.3 & 2.5 & 9.4 & 23.2 \\
\hline Student-teacher Ratio- Higher (\%) & 510 & 14.9 & 3.9 & 3.8 & 25.4 \\
\hline
\end{tabular}

Note: Ratio -Compulsory, Ratio -Senior high, Ratio - Higher are mean of the rate of student and teacher on different education level which represent the education quality of these different level. The higher of the ratio, the lower quality of the education level. Because of the 9 years compulsory education started in 1994, this paper did not calculate the education quality in the primary education

(Referring to Zhang, 2010), the human capital can be expressed by a quadratic function below:

$$
S^{*}=\mathrm{a}_{1} S+a_{2} Q+a_{3} S Q+a_{4} Q^{\wedge} 2 \log H_{i t}=S_{i t}^{*}
$$

Where $\mathrm{S}^{*}$ is the effective education; $\mathrm{S}$ is the cognitive skill measured by student-teacher ratio; 
SQ is the cross-correlation coefficient between cognitive skill and education (when the value is nonzero, the coefficient explains whether the effect of education on GDP growth depends on cognitive skill); Q^2 is the marginal effect of education level (when the value is negative, the marginal effect is declining).

\section{Methodology}

The GDP growth is often simulated by the Solow-Swan model. Here, this popular model is adopted to analyse the direct impact of education on the GDP with effective education as the exogenous variable, and to disclose the indirect effect of human capital on GDP growth. In the following analysis, H stands for human capital, Y for total GDP, K for fixed capital and L for labour force. The previous research (Papageorgiou et al., 1999) has revealed that the human capital affects GDP growth through two mechanisms: the production input and the innovation source. The two mechanisms can be explained as follows:

$$
\begin{aligned}
& Y_{\mathrm{it}}=A_{\mathrm{it}} K^{a}{ }_{i t} L_{i t}^{\beta} H^{\gamma}{ }_{i t} \\
& Y_{\mathrm{it}}=A_{\mathrm{it}}\left(H_{i t}\right) K^{a}{ }_{i t} L_{i t}^{\beta} H^{\gamma}{ }_{i t}
\end{aligned}
$$

where $A_{i t}$ are the endogenous factors of technology. These factors can be expressed as:

$$
\left(A_{\mathrm{it}}-A_{\mathrm{it}-1}\right) / A_{\mathrm{it}-1}=\delta \log H_{\mathrm{it}-1}+\mu \log H_{\mathrm{it}-1}\left(A_{\mathrm{it}-1}^{*}-A_{i t-1}\right) / A_{i t-1}
$$

where $\delta$ is the technology advancement; $\mu$ is the technology imitation; $\mathrm{A}^{*}$ is the marginal effect of technology; $\left(A_{\mathrm{it}}^{*}-A_{i t-1}\right) / A_{i t-1}$ is the technology level of the most advanced provincial entity (Shanghai) (In our research, this term is replaced by $\left.\left(y_{i t}^{*}-y_{i t-1}\right)\right)$; $\mathrm{y}^{*}$ is the per-capital output of the most advanced provincial entity.

Taking the logarithmic difference between Equations (2) and (3), we have:

$$
\log \left(Y_{\mathrm{it}} / Y_{\mathrm{it}-1}\right)=b_{0}+\alpha \log \left(K_{i t} / K_{\mathrm{it}-1}\right)+\beta \log \left(L_{i t} / L_{\mathrm{it}-1}\right)+\gamma \log \left(\mathrm{H}_{i t} / \mathrm{H}_{\mathrm{t}-1}\right)+\varepsilon_{i t}
$$

$\log \left(Y_{\mathrm{it}} / Y_{\mathrm{it}-1}\right)=b_{0}+\alpha \log H_{i t-1}+\beta\left(y_{i t}^{\prime \prime} / y_{i t}\right) \log H_{i t-1}+\gamma \log \left(K_{i t} / K_{\mathrm{it}-1}\right)+\eta \log \left(L_{i t} / L_{\mathrm{it}-1}\right)+\varphi \log \left(\mathrm{H}_{i t} / \mathrm{H}_{\mathrm{tt}-1}\right)+\varepsilon_{i t}$

Substituting $S^{*}$ into equations (5) and (6), we have:

$$
\begin{gathered}
\log \left(Y_{\mathrm{it}} / Y_{\mathrm{it}-1}\right)=b_{0}+\alpha \log \left(K_{i t} / K_{\mathrm{it}-1}\right)+\gamma_{1} \Delta S_{t-\tau}+\gamma_{2} \Delta Q_{t}+\gamma_{3} \Delta S Q_{t-\tau}+\gamma_{4} \Delta Q^{\wedge} 2_{t-\tau}+\beta \log \left(L_{i t} / L_{\mathrm{it}-1}\right)+\varepsilon_{\mathrm{it}} \\
\log \left(Y_{\mathrm{it}} / Y_{\mathrm{it}-1}\right)=b_{0}+\varphi_{1} S_{i t-1-\tau}+\varphi_{2} Q_{i t-1}+\varphi_{3} S_{i t-1-\tau} Q_{i t-1}+\varphi_{4} Q_{i t-1-\tau} \wedge 2+\left(y_{i t}^{*} / y_{i t}\right)\left(\phi_{1} S_{i t-1-\tau}+\phi_{2} Q_{i t-1}+\phi_{3} S_{i t-1-\tau} Q_{i t-1}+\phi_{4} Q_{i t-1-\tau} \wedge 2\right) \\
+\alpha \log \left(K_{i t} / K_{\mathrm{it}-1}\right)+\gamma_{1} \Delta S_{t-\tau}+\gamma_{2} \Delta Q_{t}+\gamma_{3} \Delta S Q_{t-\tau}+\gamma_{4} \Delta Q^{\wedge} 2_{t-\tau}+\beta \log \left(L_{i t} / L_{\mathrm{it}-1}\right)+\varepsilon_{\mathrm{it}}
\end{gathered}
$$


where

$$
\gamma_{1} \Delta S_{t-\tau}+\gamma_{2} \Delta Q_{t}+\gamma_{3} \Delta S_{t-\tau} \Delta Q_{t}+\gamma_{4} \Delta Q_{t-\tau} \wedge 2
$$

is the direct impact of human capital on GDP growth;

$$
\varphi_{1} S_{i t-1-\tau}+\varphi_{2} Q_{i t-1}+\varphi_{3} S_{i t-1-\tau} Q_{i t-1}+\varphi_{4} Q_{i t-1-\tau} \wedge 2
$$

Is the indirect impact of education level and cognitive skill on innovation through human capital accumulation;

$$
\left(y_{i t}^{*} / y_{i t}\right)\left(\phi_{1} S_{i t-1-\tau}+\phi_{2} Q_{i t-1}+\phi_{3} S_{i t-1-\tau} Q_{i t-1}+\phi_{4} Q_{i t-1-\tau} \wedge 2\right)
$$

Is the indirect impact of human capital on GDP growth through innovation imitation?

\section{Results and Discussion}

Based on the model of equations (5) (8), the direct and indirect impacts of human capital on economic growth were estimated and recorded in Tables $2 \sim 4$.

\begin{tabular}{|c|c|c|c|c|c|c|c|c|}
\hline \multirow{2}{*}{ Variables } & \multicolumn{4}{|c|}{ Direct effect channel-compulsory education } & \multicolumn{4}{|c|}{ Mixed effect channel- compulsory education } \\
\hline & (I) & (II) & (III) & (IV) & $(\mathrm{V})$ & $(\mathrm{VI})$ & (VII) & (VIII) \\
\hline $\log \left(K_{i t} / K_{i t-1}\right)$ & $0.014(0.91)$ & $0.014(0.94)$ & $\begin{array}{l}0.014 \\
(0.94) \\
\end{array}$ & $0.013 \quad(0.81)$ & $0.006(1.39)$ & $0.0006(1.04)$ & $0.009(1.58)$ & $\begin{array}{l}0.009 \\
(1.55) \\
\end{array}$ \\
\hline $\log \left(L_{i t} / L_{i t-1}\right)$ & $\begin{array}{c}0.097 * \\
(1.93) \\
\end{array}$ & $0.094 *(1.95)$ & $0.093 *(1.92)$ & $0.091 *(1.88)$ & $0.138 * *(2.7)$ & $0.147 *(2.96)$ & $0.140^{* *}(2.73)$ & $\begin{array}{c}0.141^{* * *} \\
(2.8)\end{array}$ \\
\hline$\Delta \mathrm{S}$ & $0.003(1.18)$ & & $\begin{array}{l}0.002 \\
(0.24)\end{array}$ & $0.005 \quad(0.46)$ & $0.006 * *(2.16)$ & & $0.015(1.16)$ & $0.022 *(1.71)$ \\
\hline$\Delta \mathcal{Q}$ & $\begin{array}{c}0.0014^{*} \\
(2.27) \\
\end{array}$ & $0.0028(1.18)$ & $\begin{array}{l}0.001 \\
(0.47) \\
\end{array}$ & $0.003 \quad(0.91)$ & $0.0008(1.65)$ & $0.004(1.94)$ & $0.003(1.05)$ & $0.007 *(1.84)$ \\
\hline$\Delta \mathrm{S} \mathcal{Q}$ & & & $\begin{array}{c}0.000006 \\
(0.02)\end{array}$ & $\begin{array}{c}-0.000007(- \\
0.22)\end{array}$ & & & $\begin{array}{r}-0.0004 \\
1.16)\end{array}$ & $\begin{array}{c}-0.0005 \\
(-1.41) \\
\end{array}$ \\
\hline$\Delta \mathcal{Q}^{2}$ & & $\begin{array}{r}-0.00002 \quad(- \\
0.75) \\
\end{array}$ & & $\begin{array}{c}-0.00002 \\
(0.79)\end{array}$ & & $\begin{array}{c}-0.00004 \\
(-1.53)\end{array}$ & & $\begin{array}{c}-0.00004 \\
(-1.26) \\
\end{array}$ \\
\hline $\mathcal{Q}_{i t-1}\left(y_{i t}^{*} / y_{i t-1}\right)$ & & & & & $\begin{array}{c}0.0005^{*} \\
(1.97)\end{array}$ & $\begin{aligned} &-0.00005(- \\
&1.85) \\
&\end{aligned}$ & $0.0005 * *(1.99)$ & $\begin{array}{c}-0.0007 \\
(-1.16) \\
\end{array}$ \\
\hline$S_{i t-1}\left(y_{i t}^{*} / y_{i t-1}\right)$ & & & & & $\begin{array}{l}-0.002 \\
(-1.18)\end{array}$ & & $\begin{array}{l}-0.003^{*} \\
(-1.76)\end{array}$ & $0.0009(0.59)$ \\
\hline$S \mathcal{Q}_{i t-1}\left(y_{i t}^{*} / y_{i t-1}\right)$ & & & & & & & $0.00004(1.25)$ & $\begin{array}{c}0.000008 \\
(0.22) \\
\end{array}$ \\
\hline $\mathcal{Q}_{i t-1}^{2}\left(y_{i t}^{*} / y_{i t-1}\right)$ & & & & & & $\begin{array}{c}0.00002 * * \\
(2.95)\end{array}$ & & $\begin{array}{c}0.00002 * * \\
(2.18)\end{array}$ \\
\hline$s_{i t-1}$ & & & & & $\begin{array}{c}0.014 * * \\
(2.43)\end{array}$ & & $0.03 * *(2.15)$ & $\begin{array}{l}0.015 \\
(0.9) \\
\end{array}$ \\
\hline $\mathcal{Q}_{i t-1}$ & & & & & $\begin{array}{r}-0.0014 \quad(- \\
1.38) \\
\end{array}$ & $0.0062 * *(2.44)$ & $0.0008(0.33)$ & $0.008 * *(2.07)$ \\
\hline$(S \mathcal{Q})_{i t-1}$ & & & & & & & $\begin{array}{r}-0.0004 \\
1.31)\end{array}$ & $\begin{array}{c}-0.0003 \\
(-0.95) \\
\end{array}$ \\
\hline $\mathcal{Q}_{i t-1}^{2}$ & & & & & & $\begin{array}{r}-0.0001^{* *} * \\
3.17)\end{array}$ & & $\begin{array}{c}-0.0001^{* *} \\
(-2.61)\end{array}$ \\
\hline _Cons & $\begin{array}{l}0.110^{* * *} \\
(31.08)\end{array}$ & $\begin{array}{l}0.111^{* * *} \\
(31.10)\end{array}$ & $\begin{array}{l}0.110^{* * * *} \\
(30.83)\end{array}$ & $\begin{array}{l}0.110^{* * *} \\
(30.72)\end{array}$ & $0.042(1.39)$ & $0.010^{* *}(3.21)$ & $\begin{array}{c}-0.047 \\
(0.5) \\
\end{array}$ & $\begin{array}{l}0.091 \\
(0.83) \\
\end{array}$ \\
\hline$N$ & 140 & 140 & 140 & 140 & 140 & 140 & 140 & 140 \\
\hline$\overline{R^{2}}$ & 0.092 & 0.085 & 0.092 & 0.097 & 0.192 & 0.211 & 0.217 & 0.271 \\
\hline
\end{tabular}

Table 2

The Different Influence Channel Based on The Compulsory Education Level 


\section{Impact of compulsory education}

The results in Table 2 show that the compulsory education has no significant impact on economic growth in either the direct effect channel or the indirect effect channel.

As shown in columns (I) (IV) of Table 2, the coefficients of education level $(\Delta \mathcal{Q})$ and cognitive skill $(\Delta \mathrm{S})$ are both insignificant, that is, the economic growth is not significantly affected by the heterogeneous human capital of compulsory education. The negative sign of $\Delta \mathcal{Q}^{2}$ indicates that the compulsory education has a decreasing marginal effect on economic growth.

The indirect effect channel of human capital in the compulsory education is described in columns (V) (VIII). In terms of technology innovation, the interaction coefficient of cognitive skill and education level $S \mathcal{Q}_{\text {it-1 }}$ was consistently negative, while the quadratic term of $\mathcal{Q}_{i t-1}^{2}$ was significantly negative. These results reveal that, when the cognitive skill is constant, the growing population receiving compulsory education will restrict independent innovation, and hinder economic growth.

In terms of innovation imitation, the education level coefficient $\mathcal{Q}_{i t-1}\left(y_{i t-1}^{*} / y_{t-1}\right)$ was insignificant. This means compulsory education level has a limited impact on economic growth through innovation imitation. Meanwhile, the interaction coefficient $S \mathcal{Q}_{i t-1}\left(y_{i t-1}^{*} / y_{t-1}\right)$ was also insignificant, indicating that the cognitive skill cannot stimulate economic growth by innovation imitation at the level of compulsory education.

To sum up, neither the education level nor cognitive skill at the compulsory education level can contribute enough human capital for economic growth. The result agrees well with that of (Liu et al., 2008).

\section{Impact of senior high school education}

The results of Table 3 show that, on the level of senior high school education, human capital accumulation through education level and cognitive skill has no significant impact on economic growth; however, the human capital does have a significant positive impact on economic growth through innovation imitation, and the impact increases with the cognitive skill improvement.

As shown in columns (I) (IV) of Table 3, the coefficient of cognitive skill $(\Delta \mathrm{S})$ was negative at the level of senior high school education. This is consistent with the previous research: cognitive skill acquired through senior high school education has no major impact on economic development, and the limited impact may be attributed to the signal effect (Barro and Salai, 1995; Prichett, 2001; Zhang et al., 2000). The negative sign of $\Delta \mathcal{Q}^{2}$ indicates that the senior high school education has a decreasing marginal effect on economic growth. Moreover, the interaction coefficient of cognitive skill and education level $(\Delta \mathrm{S} \mathcal{Q})$ was significantly positive, indicating that the population educated at senior high schools has a positive impact due to the growing accumulation of cognitive skill at this education level.

The indirect effect channel of human capital in senior high school education is described in columns (V) (VIII). In terms of technology innovation, the coefficient of education level $\left(\mathcal{Q}_{\mathrm{it}-1}\right)$ was negative and statistically significant. This means the growth of population educated at senior high schools has no major 
impact on economic growth through technology innovation. A possible reason is that the knowledge and skills of technology innovation are not taught at senior high schools. However, the interaction coefficient of cognitive skill and education level $(S Q)_{\mathrm{it}-1}$ was consistently positive, that is, the cognitive skill acquired at senior high schools can greatly enhance the self-learning and knowledge digestion abilities of the learners. At this education level, the accumulation of cognitive skill can mitigate the negative impact of education level on technology innovation.

Table 3

The Different Influence Channel Based on the Senior Education Level

\begin{tabular}{|c|c|c|c|c|c|c|c|c|}
\hline \multirow{2}{*}{ Variables } & \multicolumn{4}{|c|}{ Direct effect - Senior high education } & \multicolumn{4}{|c|}{ Mixed effect -Senior high education } \\
\hline & (I) & (II) & (III) & (IV) & $(\mathrm{V})$ & (VI) & (VII) & (VIII) \\
\hline $\log \left(K_{i t} / K_{i t-1}\right)$ & $\begin{array}{l}0.012 \\
(0.77)\end{array}$ & $\begin{array}{l}0.0125 \\
(0.83)\end{array}$ & $\begin{array}{l}0.011 \\
(0.71)\end{array}$ & $\begin{array}{l}0.011 \\
(0.71)\end{array}$ & $\begin{array}{l}0.014 \\
(0.93)\end{array}$ & $\begin{array}{l}0.004 \\
(0.83)\end{array}$ & $\begin{array}{c}-0.009 \\
(0.69)\end{array}$ & $0.015(1.14)$ \\
\hline $\log \left(L_{i t} / L_{i t-1}\right)$ & $\begin{array}{l}0.084 * \\
(1.72)\end{array}$ & $\begin{array}{l}0.063 \\
(1.29)\end{array}$ & $\begin{array}{c}0.083^{*} \\
(1.68)\end{array}$ & $\begin{array}{l}0.055^{*} \\
(1.68)\end{array}$ & $\begin{array}{l}0.079^{*} \\
(1.68)\end{array}$ & $\begin{array}{c}0.108^{* *} \\
(2.26)\end{array}$ & $\begin{array}{l}0.041 \\
(0.93)\end{array}$ & $0.048(1.12)$ \\
\hline$\Delta s$ & $\begin{array}{c}-0.004(- \\
1.52)\end{array}$ & & $\begin{array}{c}-0.011 * \\
(-1.78)\end{array}$ & $\begin{array}{l}-0.01 * \\
(-1.75)\end{array}$ & $\begin{array}{c}-0.0032 \\
(-1.17)\end{array}$ & & $\begin{array}{c}-0.016^{* * * *} \\
(-2.64)\end{array}$ & $\begin{array}{c}-0.016 * * \\
(-2.42)\end{array}$ \\
\hline$\Delta Q$ & $\begin{array}{c}0.0003 \\
(0.35)\end{array}$ & $\begin{array}{c}0.0092^{* *} \\
(3.07)\end{array}$ & $\begin{array}{l}-0.004 \\
(-1.10)\end{array}$ & $\begin{array}{l}0.004^{*} \\
(1.64)\end{array}$ & $\begin{array}{c}0.000016 \\
(0.02)\end{array}$ & $\begin{array}{c}0.012 * * * \\
(3.92)\end{array}$ & $\begin{array}{c}-0.007 * * * \\
(-2.17)\end{array}$ & $\begin{array}{l}-0.003 \\
(-0.44)\end{array}$ \\
\hline$\Delta \mathrm{S} \mathcal{Q}$ & & & $\begin{array}{l}0.0005 \\
(1.21)\end{array}$ & $\begin{array}{c}0.0005 \\
(1.46)\end{array}$ & & & $\begin{array}{c}0.00006 \\
(0.18)\end{array}$ & $\begin{array}{l}0.0002 \\
(0.55)\end{array}$ \\
\hline$\Delta Q^{2}$ & & $\begin{array}{c}-0.0002 * * \\
(-3.02) \\
\end{array}$ & & $\begin{array}{c}-0.00024 * * \\
(-2.88)\end{array}$ & & $\begin{array}{c}-0.0024 * * \\
(-2.88) \\
\end{array}$ & & $\begin{array}{c}-0.00008 \\
(-0.64) \\
\end{array}$ \\
\hline$Q_{i t-1}\left(y_{i t}^{*} / y_{i t-1}\right)$ & & & & & $\begin{array}{c}0.0014 * * \\
(3.04)\end{array}$ & $\begin{array}{c}0.0021^{* * *} \\
(1.36)\end{array}$ & $\begin{array}{c}0.0002 \\
(1.36)\end{array}$ & $\begin{array}{c}0.003 * * \\
(2.24)\end{array}$ \\
\hline$S_{i t-1}\left(y_{i t}^{*} / y_{i t-1}\right)$ & & & & & $\begin{array}{c}-0.0001^{*} \\
(-2.15)\end{array}$ & & $\begin{array}{c}-0.001 *(- \\
2.48)\end{array}$ & $\begin{array}{c}0.002 * * * \\
(3.18)\end{array}$ \\
\hline$S Q_{i t-1}\left(y_{i t}^{*} / y_{i t-1}\right)$ & & & & & & & $\begin{array}{c}0.0003 * * * \\
(4.7)\end{array}$ & $\begin{array}{c}0.0002 * * \\
(2.07)\end{array}$ \\
\hline$Q_{i t-1}^{2}\left(y_{i t}^{*} / y_{i t-1}\right)$ & & & & & & $\begin{array}{c}-0.00003 \\
(-1.00)\end{array}$ & & $\begin{array}{c}-0.00008^{*} \\
(-2.06)\end{array}$ \\
\hline$s_{i t-1}$ & & & & & $\begin{array}{c}-0.005^{*} \\
(-3.45)\end{array}$ & & $\begin{array}{c}-0.009 * * * \\
(-3.10)\end{array}$ & $\begin{array}{c}-0.017^{* * *} \\
(-2.96)\end{array}$ \\
\hline$Q_{i t-1}$ & & & & & $\begin{array}{c}0.011 * * * * \\
(4.18)\end{array}$ & $\begin{array}{c}-0.006^{*} \\
(-1.80)\end{array}$ & \begin{tabular}{|c|}
$-0.006 *(-$ \\
$1.86)$
\end{tabular} & $\begin{array}{l}0.003 \\
(0.34) \\
\end{array}$ \\
\hline$(S Q)_{i t-1}$ & & & & & & & \begin{tabular}{|c|}
0.0003 \\
$(1.04)$ \\
\end{tabular} & $\begin{array}{c}0.00007 \\
(1.21)\end{array}$ \\
\hline$Q_{i t-1}^{2}$ & & & & & & $\begin{array}{c}0.00007 \\
(0.79)\end{array}$ & & $\begin{array}{c}-0.0003 * * \\
(-2.05)\end{array}$ \\
\hline Cons & $\begin{array}{c}0.110^{* * * * *} \\
(28.89)\end{array}$ & $\begin{array}{c}0.109 * * * \\
(29.62) \\
\end{array}$ & $\begin{array}{c}0.111 \text { **** } \\
(28.7) \\
\end{array}$ & $\begin{array}{c}0.110^{* * * *} \\
(24.49)\end{array}$ & $\begin{array}{c}0.101 \text { *** } \\
(2.8) \\
\end{array}$ & $\begin{array}{c}0.104 * * * \\
(3.9) \\
\end{array}$ & $\begin{array}{c}0.192 * * * * \\
(3.59) \\
\end{array}$ & $\begin{array}{c}0.174 * * \\
(2.39) \\
\end{array}$ \\
\hline $\mathrm{N}$ & 140 & 140 & 140 & 140 & 140 & 140 & 140 & 140 \\
\hline R2 & 0.059 & 0.106 & 0.072 & 0.139 & 0.285 & 0.245 & 0.428 & 0.466 \\
\hline
\end{tabular}

Note. *,**,*** Denote significance at the $10 \%, 5 \%$ and $1 \%$ levels, respectively;()is the $\mathrm{z}$ value of each coefficient of the explanatory variable.

In terms of innovation imitation, the education level coefficient Q_(it-1) (y_(it-1)^*/y_(it-1) ) was significantly positive. This means the length of senior high school education has a positive impact on innovation imitation. Meanwhile, the interaction coefficient [SQ]_(it-1) (y_(it-1)^*/y_(it-1) ) between education level and cognitive skill was significant, indicating that the cognitive skill acquired at senior high schools can further enhance the positive impact on innovation imitation. Nevertheless, the negative sign of the quadratic education year coefficient Q_(it-1)^2 (y_(it-1)^*/y_(it-1) ) implies that the marginal effect of senior high school education on the economic growth gradually decreases through innovation imitation. 
In short, the education level and cognitive skill at the level of senior high schools have decreasing positive impacts on economic growth through innovation imitation, and the accumulation of cognitive skill can mitigate the negative impact of education level on technology innovation. In the long run, however, it is impossible to promote economic growth effectively by expanding the education level at this education level, even if the cognitive skill is enhanced at the same time.

\begin{tabular}{|c|c|c|c|c|c|c|c|c|}
\hline \multicolumn{9}{|c|}{$\begin{array}{l}\text { Table } 4 \\
\text { The Different Influence Channel Based on the Higher Education Level }\end{array}$} \\
\hline \multirow{2}{*}{ Variables } & \multicolumn{4}{|c|}{ Direct effect channel-higher education } & \multicolumn{4}{|c|}{ Mixed effect channel-higher school } \\
\hline & (I) & (II) & (III) & (IV) & $(\mathrm{V})$ & $(\mathrm{VI})$ & (VII) & (VIII) \\
\hline $\log \left(K_{i t} / K_{i t-1}\right)$ & $\begin{array}{c}0.0034 \\
(1.21) \\
\end{array}$ & $\begin{array}{l}0.0185 \\
(1.23) \\
\end{array}$ & $\begin{array}{l}0.0001 \\
(0.89) \\
\end{array}$ & $\begin{array}{l}0.005 \\
(1.5)\end{array}$ & $\begin{array}{l}0.002 \\
(1.15)\end{array}$ & $\begin{array}{l}0.001 \\
(1.39) \\
\end{array}$ & $\begin{array}{l}0.004 \\
(1.26) \\
\end{array}$ & $\begin{array}{l}0.002 \\
(1.15) \\
\end{array}$ \\
\hline $\log \left(L_{i t} / L_{i t-1}\right)$ & $\begin{array}{l}0.098^{*} \\
(2.02) \\
\end{array}$ & $\begin{array}{l}0.071 \\
(1.47) \\
\end{array}$ & $\begin{array}{l}0.095^{*} \\
(1.99) \\
\end{array}$ & $\begin{array}{c}0.081^{*} \\
(1.7) \\
\end{array}$ & $\begin{array}{c}0.091^{* *} \\
(1.94) \\
\end{array}$ & $\begin{array}{c}0.100^{* * *} \\
(2.31) \\
\end{array}$ & $\begin{array}{l}0.077^{*} \\
(1.68)\end{array}$ & $\begin{array}{l}0.076^{*} \\
(1.67)\end{array}$ \\
\hline$\Delta S$ & $\begin{array}{c}0.0034^{*} \\
(2.21)\end{array}$ & & $\begin{array}{c}-0.0011 \\
(-0.45) \\
\end{array}$ & $\begin{array}{c}-0.0016 \\
(-0.65) \\
\end{array}$ & $\begin{array}{c}0.0013 \\
(0.66) \\
\end{array}$ & & $\begin{array}{l}0.0014 \\
(0.54)\end{array}$ & $\begin{array}{c}-0.0014 \\
(-0.47) \\
\end{array}$ \\
\hline$\Delta Q$ & $\begin{array}{c}0.0008 \\
(1.33) \\
\end{array}$ & $\begin{array}{c}0.0031^{* *} \\
(2.91)\end{array}$ & $\begin{array}{r}-0.0027 \\
(-1.61) \\
\end{array}$ & $\begin{array}{c}-0.0007 \\
(-1.39) \\
\end{array}$ & $\begin{array}{c}0.0013^{* *} \\
(2.04)\end{array}$ & $\begin{array}{c}0.003 * * \\
(2.31) \\
\end{array}$ & $\begin{array}{c}0.0022 \\
(1.16) \\
\end{array}$ & $\begin{array}{c}0.0007 \\
(0.29) \\
\end{array}$ \\
\hline$\Delta \mathrm{S} Q$ & & & $\begin{array}{c}0.0003 * \\
(2.29) \\
\end{array}$ & $\begin{array}{c}0.0003^{*} \\
(2.23) \\
\end{array}$ & & & $\begin{array}{c}-0.0003^{* *} \\
(-1.98)\end{array}$ & $\begin{array}{c}-0.00006 \\
(-0.29)\end{array}$ \\
\hline$\Delta \mathcal{Q}^{2}$ & & $\begin{array}{c}-0.00006^{* *} * \\
(-2.57) \\
\end{array}$ & & $\begin{array}{c}-0.00005^{*} \\
(-2.13)\end{array}$ & & $\begin{array}{c}-0.00005^{* *} \\
(-2.40) \\
\end{array}$ & & $\begin{array}{c}-0.00002 \\
(-0.92) \\
\end{array}$ \\
\hline $\mathcal{Q}_{i t-1}\left(y_{i t}^{*} / y_{i t-1}\right)$ & & & & & $\begin{array}{c}0.001 * * * \\
(3.41) \\
\end{array}$ & $\begin{array}{c}0.002 * * \\
(3.34) \\
\end{array}$ & $\begin{array}{c}0.0008^{* *} \\
(2.49)\end{array}$ & $\begin{array}{c}0.0015^{* * *} \\
(2.22)\end{array}$ \\
\hline$S_{i t-1}\left(y_{i t}^{*} / y_{i t-1}\right)$ & & & & & $\begin{array}{c}-0.0002 \\
(-1.28) \\
\end{array}$ & & $\begin{array}{c}-0.0004^{*} \\
(-1.85) \\
\end{array}$ & $\begin{array}{c}-0.0003 \\
(-1.46) \\
\end{array}$ \\
\hline$S Q_{i t-1}\left(y_{i t}^{*} / y_{i t-1}\right)$ & & & & & & & $\begin{array}{c}0.00008^{* *} \\
(2.94) \\
\end{array}$ & $\begin{array}{c}0.00005^{*} \\
(1.69)\end{array}$ \\
\hline $\mathcal{Q}_{i t-1}^{2}\left(y_{i t}^{*} / y_{i t-1}\right)$ & & & & & & $\begin{array}{c}-0.00002 \\
(-0.75) \\
\end{array}$ & & $\begin{array}{c}-0.00002 \\
(-0.61) \\
\end{array}$ \\
\hline$S_{i t-1}$ & & & & & $\begin{array}{l}0.003 * * \\
(3.11)\end{array}$ & & $\begin{array}{c}0.003^{* *} \\
(1.91)\end{array}$ & $\begin{array}{l}0.006^{*} \\
(2.43)\end{array}$ \\
\hline$Q_{i t-1}$ & & & & & $\begin{array}{c}0.003^{*} \\
(1.72) \\
\end{array}$ & $\begin{array}{c}0.0071^{* * *} * \\
(5.26) \\
\end{array}$ & $0.003(1.22)$ & $\begin{array}{c}0.0016 \\
(0.50) \\
\end{array}$ \\
\hline$(S Q)_{i t-1}$ & & & & & & & $\begin{array}{c}-0.00022^{*} \\
(-1.67)\end{array}$ & $\begin{array}{c}-0.00009 \\
(-1.58)\end{array}$ \\
\hline$Q_{i t-1}^{2}$ & & & & & & $\begin{array}{c}0.00008 \\
(1.52)\end{array}$ & & $\begin{array}{c}0.00007 \\
(1.24)\end{array}$ \\
\hline Cons & $\begin{array}{c}0.115 * * * \\
(24.56) \\
\end{array}$ & $\begin{array}{c}0.108 * * * \\
(24.48) \\
\end{array}$ & $\begin{array}{c}0.115 * * * \\
(24.71) \\
\end{array}$ & $\begin{array}{c}0.113 * * * \\
(24.12) \\
\end{array}$ & $\begin{array}{c}0.087 * * * \\
(3.4)\end{array}$ & $\begin{array}{c}0.124^{* * * *} \\
(12.50) \\
\end{array}$ & $\begin{array}{c}0.0922 * * \\
(2.89) \\
\end{array}$ & $\begin{array}{c}0.114 * * \\
(2.84) \\
\end{array}$ \\
\hline $\mathrm{N}$ & 140 & 140 & 140 & 140 & 140 & 140 & 140 & 140 \\
\hline $\mathrm{R} 2$ & 0.095 & 0.108 & 0.137 & 0.172 & 0.295 & 0.341 & 0.357 & 0.382 \\
\hline
\end{tabular}

\section{The İmpact of Higher Education}

The results in Table 4 shows that human capital acquired in higher education can boost economic growth through various channels. The human capital exerts a significant positive effect on economic growth directly as a production input, and indirectly through technology innovation and innovation imitation. The education level of higher education has a slightly lower impact on innovation imitation than that in senior high school education, which may be attributed to the small proportion of people receiving higher education. 
As shown in columns (I) (IV) of Table 4, the interaction term between education level and the cognitive skill was significantly positive, and the quadratic education level coefficient $\Delta \mathcal{Q}^{2}$ was much smaller than that the level of senior high school education.

These results suggest that the population receiving higher education directly bears on the economic growth, and the impact is a linear positive one. This impact can be explained by the small proportion of the population receiving higher education in the total labour force.

The indirect effect channel of human capital in higher education is described in columns (V) (VIII). In terms of technology innovation, the coefficient of education level $\left(\Delta \mathcal{Q}_{i t-1}\right)$ was significantly positive. This means the growth of population receiving higher education can accelerate the economic growth by technology innovation. The negative sign of the $(S \mathcal{Q})_{i t-1}$ implies that, when the cognitive skill is constant, the excessive growth in the population receiving higher education will restrict independent innovation.

In terms of innovation imitation, the $\mathcal{Q}_{i t-1}\left(y_{i t}^{*} / y_{i t-1}\right)$ and $(S \mathcal{Q})_{i t-1}$ of higher education were compared with those of senior high school education. The results show that, when the cognitive skill is constant, the positive impact from the population educated at senior high schools on GDP growth is three times that from the population receiving higher education. In other words, the senior high school education boasts a greater impact on economic growth through innovation imitation than higher education.

\section{Conclusion}

The following conclusions were drawn from the analysis on the effect channels of education level and cognitive skill at different education levels on economic growth.

On the compulsory education level, neither educated populated nor cognitive skill has any direct or indirect impact on economic growth. The growth in the population receiving primary education will suppress technology innovation and innovation imitation.

On the level of senior high school education, the education level has a significant positive effect on economic growth indirectly through innovation imitation. This impact can be further enhanced by the improvement of cognitive skill.

On the higher education level, the human capital exerts a greater positive effect on economic growth directly as a production input than that of compulsory and senior high school educations. Note that the indirect impact on economic growth through technology innovation can also be bolstered by the improvement of cognitive skill. In addition, the senior high school education boasts a greater indirection impact on economic growth through innovation imitation than higher education.

Therefore, it is possible to stimulate economic growth by decreasing the proportion of labour force receiving compulsory education. The improvement of the cognitive skill of the labour force at different education levels is key to both technology innovation and innovation imitation. Besides, the human capital structure should be optimized by simultaneous improvement of education level and cognitive skill. 


\section{References}

Aghion, P., Howitt, P., Howitt, P. W., Brant-Collett M, \& García-Peñalosa C. (1998). Endogenous growth theory. MIT press, https://dx.doi.org/10.1057/9780230280823_10.

Barro, R. (1991). Economic growth in a cross-section of countries. The Quarterly Journal of Economics, 106(2), 407-443, https:/dx./doi.org/10.1111/1467-9361.00204.

Breton, T. R. (2011). The quality vs. the quantity of schooling: What drives economic growth. Economics of Education Review, 30(4), 765-773, https://doi.org/10.1016/j.econedurev.2011.01.005.

Burdett, K. B., Melvyn, G. C. (1997). Marriage and class. Quarterly Journal of Economics, 112(1), 141-168, https://dx.doi.org/10.1162/003355397555154.

Griliches, Z. (1979). Issues in assessing the contribution of R\&D to productivity. Bell Journal of Economics, 10, 92-116, https://dx.doi.org/10.2307/3003321

Hanushek, E. A., \& Kimko, D. D. (2000). Schooling, labor-force quality, and the growth of nations. American Economic Review, 90(5), 1184-1208, https://dx.doi.org/10.2307/2677847.

Hanushek, E. A., \& Woessmann, L. (2008), The role of cognitive skills in economic development. Journal of Economic Literature, 46(3), 607-608, https://dx.doi.org/10.2307/27647039.

Hsieh, C. T., Klenow, P. J. (2009). Misallocation and manufacturing TFP in China and India. The Quarterly Journal of Economics, 124(4), 1403-1448, https://dx.doi.org/10.1162/qjec.2009.124.4.1403.

Hu. A., Hibel, J. (2015). Increasing heterogeneity in the economic returns to higher education in urban China. The Social Science Journal, 52(3), 322-330, https://dx.doi.org/10.1016/j.soscij.2013.09.002.

Jovanovic, B. (2014). Misallocation and growth. American Economic Review, 104(4), 1149-71, https://dx.doi.org/10.1257/aer.104.4.1149.

Liu, Z. Y., Hu, Y. Y., \& Yi, X. R. (2008). Testing for the mechanism of impacts of heterogeneous human capital on economic growth. The Journal of Quantitative and Technical Economics, (4), 86-96.

Lucas-Jr, R. E. (1988). On the mechanics of economic development. Journal of Monetary Economics 22(1), 342, https://dx.doi.org/10.1016/0304-3932(88)90168-7.

Midrigan, V., \& Xu, D. Y. (2014). Finance and misallocation: Evidence from plant-level data. American Economic Review, 104(2): 455-58, https://dx.doi.org/10.1257/aer.104.2.422.

Paganetto, L., \& Scandizzo, P. L. (2003). The role of education and knowledge in endogenous growth. In Finance, Research, Education and Growth. London: Palgrave Macmillan, 90-104, https://dx.doi.org/10.1057/9781403920232_6.

Qian, X., Chi, W., Li, B. (2010). Human capital has effect on regional innovation and Economic growthspatial regression model. Mathematic economic technology research, 4, 107-20

Restuccia, D., \& Rogerson, R. (2008), Policy distortions and aggregate productivity with heterogeneous establishments. Review of Economic dynamics, 11(4), 707-20, https://dx.doi.org/10.1016/j.red.2008.05.002.

Zhang, H. F., \& Yao, X. B. (2010). Zhang JS. The influence of education quality for regional labor productivity. Journal of economic research, 7, 57-66. 